\title{
Performance evaluation of new blind OFDM signal recognition based on properties of the second-order statistics using universal software radio peripheral platform
}

\author{
Mohamed Firdaoussi ${ }^{1}$, Hicham Ghennioui ${ }^{2}$, Mohamed El Kamili ${ }^{3}$, Mohamed Lamrini ${ }^{4}$ \\ ${ }^{1,4}$ LPAIS, Faculty of Sciences Dhar El Mahraz, Sidi Mohamed Ben Abdellah University of Fez, Morocco \\ ${ }^{2}$ LSSC, Faculty of Sciences and Technologies, Sidi Mohamed Ben Abdellah University of Fez, Morocco \\ ${ }^{3} \mathrm{C} 3 \mathrm{~S}$, Higher School of Technology, Hassan II University of Casablanca, Morocco
}

\section{Article Info}

Article history:

Received Dec 13, 2020

Revised Jun 25, 2021

Accepted Jul 3, 2021

\section{Keywords:}

Cognitive radio

MATLAB

Modulation recognition

OFDM

Second-order statistics

SNR

USRP

\begin{abstract}
In the context of cognitive radio (CR) or various military and civilian applications, modulation recognition (MR) is one of the most popular technical processes in the field of communication system recognition, by which the modulation type of the unknown received signal can be identified automatically by estimating one or more parameters of the modulated signal. This paper presents the performance evaluation of the new proposed blind system recognition method using only a particular property of the secondorder statistics of the orthogonal frequency division multiplexing (OFDM) modulated signal. The effectiveness of the proposed method is illustrated using the implementation on universal software radio peripheral (USRP) platform. A comparison with computer simulations using MATLAB software is also performed, emphasizing the good performances of the method while the results obtained are close. We show the efficiency and behavior of the proposed method in the context of wireless communication systems based on OFDM modulation (3GPP/LTE, WiMAX, DVBT-2K, IEEE 802.22-1K, IEEE 802.22-2K, IEEE 802.22-4K). The proposed method can detect OFDM signals among other digital signals in a systematic and intelligent way even with low SNR values (when approaching to $S N R=-2 \mathrm{~dB}$, the decision criteria tends towards 0 ).
\end{abstract}

This is an open access article under the CC BY-SA license.

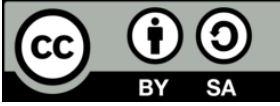

\section{Corresponding Author:}

Mohamed Firdaoussi

Laboratoire de Physique Appliquée, Informatique et Statisitique (LPAIS)

Faculté des Sciences Dhar El Mahraz de Fès (FSDM)

Université Sidi Mohamed Ben Abdellah, B.P. 1796 Fès-Atlas, 30003, Maroc

Email: mohamed.firdaoussi@usmba.ac.ma

\section{INTRODUCTION}

Digital communication systems have undergone rapid development in recent years, prompting scientists and innovators to conceive new antennas [1] and more advanced digital communication techniques to increase the effective capacity of wireless systems. Among these techniques, modulation recognition (MR) is one of the most widespread techniques and plays an influential role in a diversity of civilian and military applications, particularly in the monitoring and control of broadcasting activities, radar system, electronic warfare, spectrum monitoring, management and cognitive radio. However, the only problem was the almost total occupation of the spectral resource managed by regulatory agencies. It has been noticed that many frequencies are unoccupied during the day when they are officially allocated to primary systems such as television (TV) [2] and could be used by secondary wireless systems such as 3GPP/LTE, WiMAX, Wi-Fi, as 
a good optimization to use the spectrum resource. Hence, the concept of cognitive radio (CR) [3], [4] is considered as an approach to be exploited for dynamic spectrum access implementation and which allows us to avoid interference with primary users. This is why cognitive radio has been considered as a key solution for the future of wireless communication systems by having the ability to intelligently detect the environment and autonomously adapt its transmission so as not to interfere with the main users. Different Spectrum Sensing detection techniques [5]-[10] have been developed and used to extract information. In practice, it means that it is necessary to know the current type of standard before classifying it on each frequency band on which it could communicate with the receiver. Nevertheless, most of the current systems are based on digital modulation techniques such as OFDM, and the wireless industry expressed a high interest in OFDM technology, due to the following benefits of OFDM, including optimal use of the allocated frequency band, high-speed data transmission, elimination of point noise phenomena, immunity to multipath fading and simplicity of equalization [11], [12]. The OFDM technique has been integrated in a variety of applications and standards, such as IEEE 802.11a [13] and IEEE 802.16a [14]. In addition, the burgeoning OFDM wireless communication technology presents a new challenge to smart radio designers, which is the recognition of digital systems based on OFDM multi-carrier modulation. The methods studied in [15]-[18] have made it possible to classify automatically the digital modulations without a priori knowledge of the parameters of the received signal by forced estimation of the signal and noise power, carrier frequency recovery and the recovery of symbol timing and carrier information, respectively. Most of the proposed algorithms are based on signal models cyclostationarity [19]-[26]. Some of them used the cyclic prefix (CP) as a parameter inducing cyclic statistics obtained by the properties of the autocorrelation function [19]-[27]. There are also others that require the detection of cyclostationary signatures as a feature consciously embedded in digital communication signals by focusing on OFDM and represented as a unique identifier [22] by sending redundant message symbols on multiple subcarriers, on the other hand the pilot-induced cyclic statistics have been reported in [22].

This paper proposes a new method based only on particular properties of the second-order statistics that characterizes the properties of the received signal as a measuring instrument. In other words, it is to define a new decision criterion that gives us an optimal solution by which systems based on OFDM modulation can be distinguished from other modulation types. The recognition performance of the proposed algorithm is implemented through an additive white Gaussian noise (AWGN) channel into numerical computations using MATLAB and experimental measurements using NI USRP hardware devices and NI LabVIEW platform. The main objective of this work is then to evaluate the performance of the proposed method in a context close to reality.

The rest of this paper is organized as follows. OFDM signal model and its recognition approach based on the correlation function are introduced in section 2. Implementation details using NI USRP-2930 are presented in section 3. Simulation and experimental results of the implementation process are given and explained in section 4 , and conclusions are drawn in section 5.

\section{SIGNAL MODEL AND SECOND-ORDER STATISTICS}

In this section, we present briefly the definition of the transmitted and received signal for multicarrier modulation (OFDM), in addition to the approach used under the second-order statistics properties.

\subsection{OFDM signal model}

The transmitted continuous-time OFDM signal is written as follows [19]:

$$
x_{O F D M}(t)=\frac{1}{\sqrt{N}} \sum_{k=0}^{K-1} \sum_{n=0}^{N-1} a_{k, n} e^{-2 i \pi \frac{n\left(t-D T_{c}-k T_{S}\right)}{N T_{C}}} g^{\operatorname{tr}}\left(t-k T_{S}\right)
$$

where $\left\{a_{k, n}\right\}$ represents the data symbols of the unknown information data of subcarrier $n$ and $k$ OFDM block and which are assumed to be zero-mean and be independent and identically distributed random variables. $K$ is the number of OFDM symbols, $N$ is the number of subcarriers and $T_{c}$ is the chip duration where $1 / T_{c}$ represents the information symbol rate in the absence of guard interval. $N T_{c}$ is the intercarrier spacing(useful part of OFDM signal). $D T_{c}$ represents the length of the cyclic prefix, $T_{s}=(N+D) T_{c}$ is the OFDM symbol interval (total duration), and $g^{t r}(t)$ is the overall impulse response of the transmit filter that is assumed to be equal to 1 if $t \in\left[0, T_{s}\right]$ and 0 otherwise.

At the receive-side, the continuous-time baseband OFDM signal equivalent can be represented as: 


$$
\begin{aligned}
r_{O F D M}(t) & =\frac{e^{2 i \pi \delta f t}}{\sqrt{N}} \sum_{l=1}^{L} \sum_{k=0}^{K-1} \sum_{n=0}^{N-1} \lambda_{l} e^{2 i \pi n \frac{\tau_{l}}{N T_{c}}} a_{k, n} e^{-2 i \pi \frac{n\left(t-D T_{c}-k T_{S}\right)}{N T_{c}}} g\left(t-\tau_{l}-k T_{S}\right) \\
& +\omega(t),
\end{aligned}
$$

where $L$ is the number of paths performed when the transmitted signal passes through a multipath fading channel. The amplitude and the delay of the $l^{\text {th }}$ path are respectively denoted by $\lambda_{l}$ and $\tau_{l}$. $\omega(t)$ is a zero-mean complex Gaussian noise with variance $\sigma_{n}^{2}$ and where $\delta f$ is the offset frequency due to local oscillator drift or Doppler effect [19]. The received continuous time OFDM signal $r_{O F D M}(t)$ is sampled at the sampling frequency $F_{e}=1 / T_{e}$ and $T_{e}$ is the sampling period. Let $M=\left\lfloor T_{0} / T_{e}\right\rfloor$ the number of samples received where $T_{0}$ is the duration of the observation window and [.] represents the integer part operator. The discrete-time OFDM received signal is denoted by $r_{O F D M}[m]=r_{O F D M}\left(m T_{e}\right)$ and is written as:

$$
\begin{aligned}
r_{O F D M}[m] & =\frac{1}{\sqrt{N}} \sum_{l=1}^{L} \sum_{k=0}^{K-1} \sum_{n=0}^{N-1} \lambda_{l} e^{2 i \pi n \frac{\tau_{l}}{N T_{c}}} a_{k, n} e^{-2 i \pi n m \frac{T_{e}}{N T_{c}}} e^{2 i \pi(k+1) \frac{D T_{c}}{N T_{c}}} g^{\operatorname{tr}}\left(m T_{e}-\tau_{l}-k(N+D) T_{c}\right) \\
& \times e^{2 i \pi \Delta f m}+W[m],
\end{aligned}
$$

with $W[m]=\omega\left(m T_{e}\right)$, and $\Delta f=\delta f T_{e}$ the normalized carrier frequency offset.

\subsection{Recognition of OFDM systems approach}

In this section, we present a proposed blind method for recognition of OFDM signal using only a proprietie of the second-order statistics as shown in Figure 1 in order to define the decision criteria which lead us to quickly recognize the OFDM signal against other signal types, in the context of Gaussian channel and perfect time and frequency synchronization $\left(L=1 ; \lambda_{l}=1 ; \tau_{l}=0 ; \delta f=0\right)$. The correlation function of the discrete-time OFDM received signal described in (3) can be expressed as (4):

$$
\begin{aligned}
& C_{r}^{O F D M}[m, p]=\mathbb{E} \llbracket r_{O F D M}[m] r_{O F D M}^{*}[m-p] \rrbracket= \\
& \frac{\sigma_{a}^{2}}{N} \sum_{k=0}^{K-1} \sum_{n=0}^{N-1} e^{-i 2 \pi n \frac{T_{e}}{N T_{c}} p} C_{g}^{t r}\left(m-k \frac{T_{s}}{T_{e}}, p\right)+\sigma_{n}^{2} \delta[p],
\end{aligned}
$$

where the superscript (.)* means the conjugate operator, $\sigma_{a}^{2}=\mathbb{E}\left|a_{k, n}\right|^{2}$ is the variance of the symbol $a_{k, n}$,

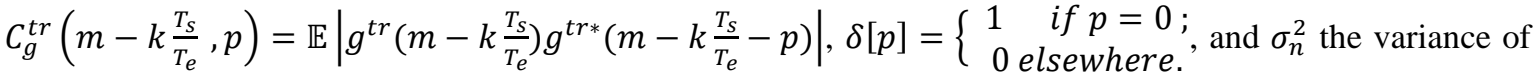
the Gaussian noise which is written as (5):

$$
\sigma_{n}^{2}=\frac{T_{c}}{T_{e}} \frac{1}{M} \sum_{m=0}^{M-1}\left|\sum_{l=1}^{L} \lambda_{l} x_{O F D M}\left(m T_{e}-\tau_{l}\right)\right|^{2} 10^{-\frac{S N R}{10}}
$$

The mean correlation function is written as (6):

$$
\begin{aligned}
& C_{r_{O F D M}}[p]=\sum_{m} C_{r}^{O F D M}[m, p]=\sum_{k=0}^{K-1} C_{r}^{O F D M}\left[k T_{s}\right] \delta\left[p-k T_{s}\right] \\
& +C_{r}^{O F D M}\left[(k+1) T_{u}\right] \delta\left[p-(k+1) T_{u}\right]+C_{r}^{O F D M}\left[-(k+1) T_{u}\right] \delta\left[p+(k+1) T_{u}\right]
\end{aligned}
$$

where $T_{u}=\frac{N T_{c}}{T_{e}}$ denotes the number of samples in the useful part of the OFDM symbol.

For $k$ block of an OFDM signal, the elementary correlation function can be expressed as (7):

$$
\begin{aligned}
& C_{r_{O F D M}}^{(k)}[p]=C_{r}^{O F D M}\left[k T_{s}\right] \delta\left[p-k T_{s}\right]+C_{r}^{O F D M}\left[(k+1) T_{u}\right] \delta\left[p-(k+1) T_{u}\right] \\
& +C_{r}^{O F D M}\left[-(k+1) T_{u}\right] \delta\left[p+(k+1) T_{u}\right]
\end{aligned}
$$

In Figure 1, the mean correlation function $C_{r_{O F D M}}[p]$ of all considered systems (3GPP(LTE), WiMAX (IEEE 802.16), DVB-T 2K, IEEE 802.22-1K, IEEE 802.22-2K, IEEE 802.22-4K) is displayed. 

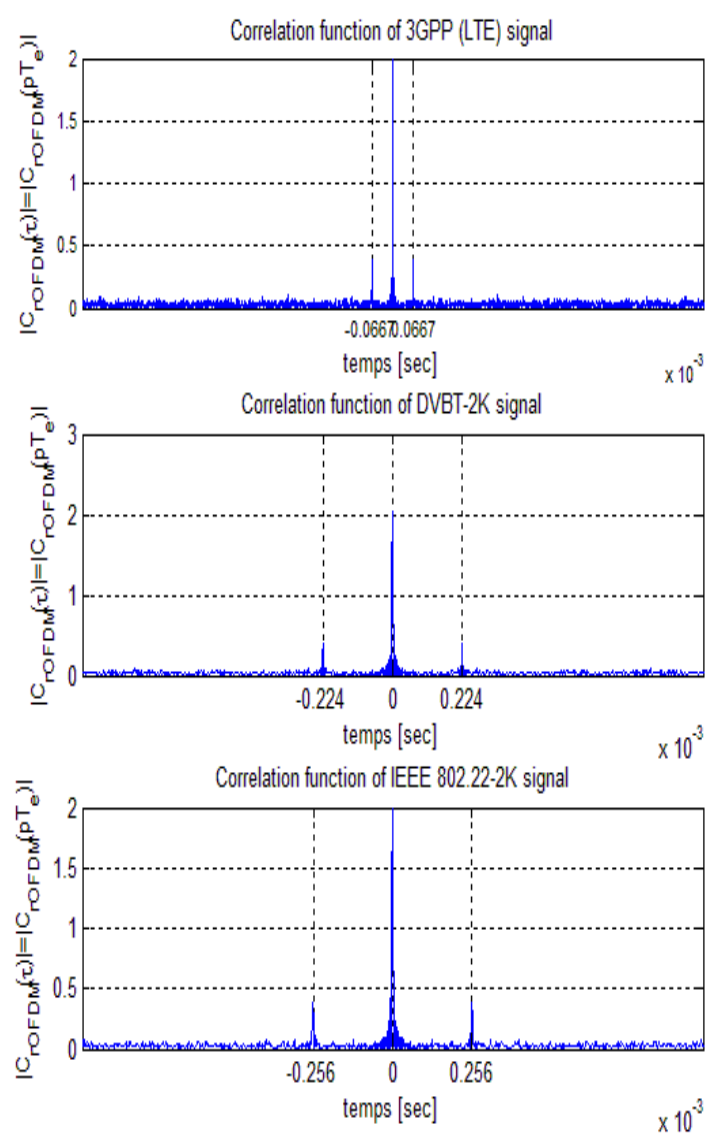
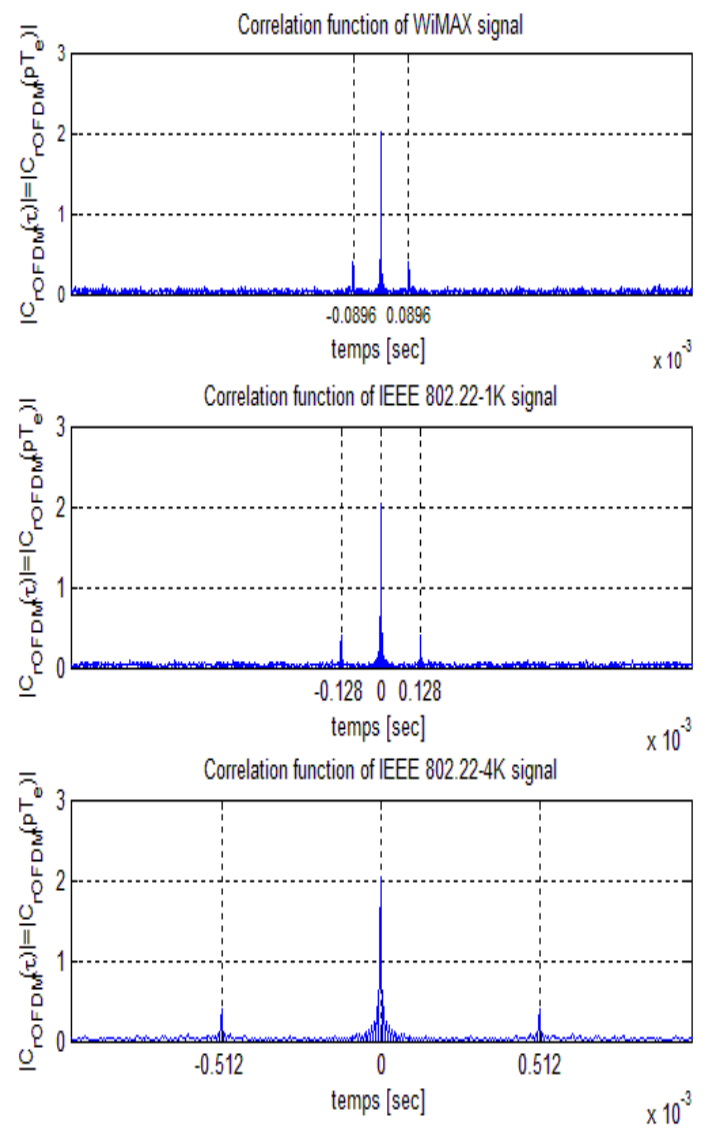

Figure 1. The modulus of mean correlation function of 3GPP (LTE), WiMAX (IEEE 802.16), DVB-T 2K, IEEE $802.22-1 \mathrm{~K}$, IEEE 802.22-2K, and IEEE 802.22-4K

It is clear from this figure, the correlation function possesses a peak at $\tau=\mathrm{T}_{\mathrm{u}}=\mathrm{NT}_{\mathrm{c}}$ (the useful part of OFDM signal): We found $6.666 .10^{-5}$ (resp. 8.96.10-5 $2.24 .10^{-4}, 1.28 .10^{-4}, 2.56 .10^{-4}$ and $5.12 .10^{-4}$ ) for 3GPP (resp. WiMAX, DVBT-2K, IEEE 802.22-1K, IEEE 802.22-2K, and IEEE 802.22-4K). We exploit this feature to propose a new method of an OFDM-based system recognition. The recognition of OFDM relative to other modulated digital signals can be formulated by $\xi$ (defined as the geometric over the arithmetic mean of the sequence $|f(k)|)$ :

$$
\xi=\frac{\left(\prod_{k=0}^{K-1}|f(k)|^{\gamma^{k}}\right)^{\frac{1}{\Gamma}}}{\frac{1}{\Gamma} \sum_{k=0}^{K-1} \gamma^{k}|f(k)|}, \Gamma=\sum_{k=0}^{K-1} \gamma^{k}=\frac{1-\gamma^{K}}{1-\gamma}
$$

with $f(k)=\max _{p>0}\left|C_{r_{O F D M}}^{(k)}[p]\right|^{2}, 0 \leq \gamma \leq 1$, and $\xi$ can be shown that:

$$
\left\{\begin{array}{l}
0 \leq \xi \leq 1 \\
\xi=1 \Leftrightarrow|f(k)|=\left|f\left(k^{\prime}\right)\right| \forall k, k^{\prime}
\end{array}\right.
$$

The Decision criteria can be then expressed as (9):

$$
D_{c}=|1-\xi| \stackrel{O F D M}{\lessgtr} \eta,
$$

where $\eta$ is the predefined decision threshold for the OFDM and other modulated digital signals, and is given by [10]: 


$$
\eta=\sigma_{n}^{2}\left(\frac{Q^{-1}\left(P_{f a}\right)}{\sqrt{S}}+1\right)
$$

with $\sigma_{n}^{2}$ is the variance of $A W G N$ channel, $P_{f a}$ is the false alarm probability targeted by $\eta, S$ corresponds to the length of the observation sequence of signal, and $Q^{-1}($.$) is the inverse Gaussian Q$-function. Then the block-diagram for the modulation recognition of OFDM signal is shown in Figure 2.

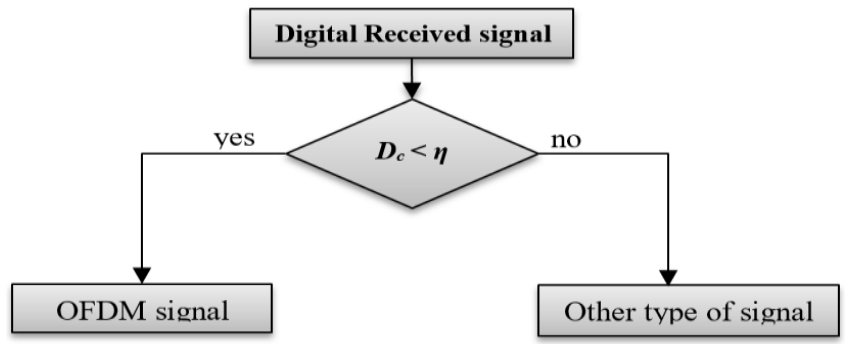

Figure 2. Block-diagram of proposed recognition system

\section{IMPLEMENTATION ENVIRONMENT}

In this section, we used as an implementation environment a software defined radio (SDR) platform in form of a NI USRP-2930 prototype able to transmit and receive radio frequency (RF) signals across a real time, paired with NI labVIEW 2017 and MATLAB R2016b software which are installed on a desktop computer, and connected through a RJ45 Gigabit Ethernet cable as shown in Figure 3.

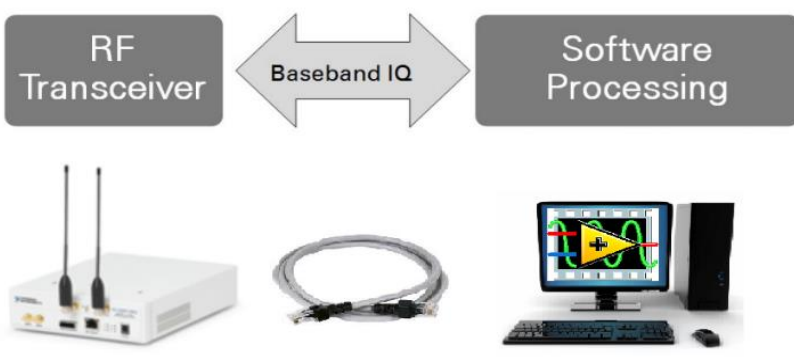

Figure 3. Simplified Overview of a SDR Setup Built Around an NI USRP-2930

\subsection{NI USRP-2930}

The USRP-2930 is a tunable radio frequency (RF) transceiver with a high-speed analog-to-digital converter and digital-to-analog converter for streaming baseband I and Q signals to a host PC over 1 Gigabit Ethernet. The NI USRP-2930 model enables to transmit and receive RF signals across a frequency range from $50 \mathrm{MHz}$ up to $2.2 \mathrm{GHz}$ with an instantaneous Real-Time bandwidth of $20 \mathrm{MHz}$ (with 16-bit samples width) or $40 \mathrm{MHz}$ (with 8-bit samples width), and it gives us the ability to use it in the following communications applications such as: broadcast FM; low-power unlicensed devices on industrial, scientific, and medical (ISM) bands; cell phone; GPS. It also included GPS-Disciplined oscillator (GPSDO) with PPS accuracy of $\pm 50 \mathrm{~ns}[28]$.

\subsection{Transmitter/receiver}

The programming and design are realized in LabVIEW 2017 in order to control the NI USRP-2930 hardware. In Figure 4, the front panel of the transmitter VI contains two parts, the left part is composed of two tabs, one for the parameters of the USRP, input parameters of the generated signal to be transmitted, and the second tab "Debug" for the errors detected during the sending operation, the second part displays the power spectrum model of OFDM signal transmitted. All baseband I/Q transmitted signals expressed in samples per second (S/s) are synthesized by the host computer and fed to the USRP-2930 at up to $400 \mathrm{KS} / \mathrm{s}$ over Gigabit Ethernet when represented with 16-bits (8-bits each for the I and Q components). The resulting analog signals are then mixed up to $200 \mathrm{MHz}$ carrier frequency. 
At the receive-side, the front panel of the receiver VI as shown in Figure 5, has two parts, the left part contains two tabs, the first tab is especially for the USRP settings which are the same as the transmitter parameters except that the antenna is set to RX2, in addition to the results obtained by our method such as the value of the decision criteria $\left(D_{c}\right)$, and the detection probability $\left(P_{D}\right)$ depending on the False-alarm Probability $\left(P_{f a}\right)$, the value of the Signal-to-noise ratio $(S N R)$ and number of realizations, the second tab "Debug" displays the errors detected during reception. The right part of the front panel displays the power spectrum model of the received OFDM signal with a certain noise level added at the top (red color, SNR= $-10 \mathrm{~dB}$ ) and without noise at the bottom (white color).

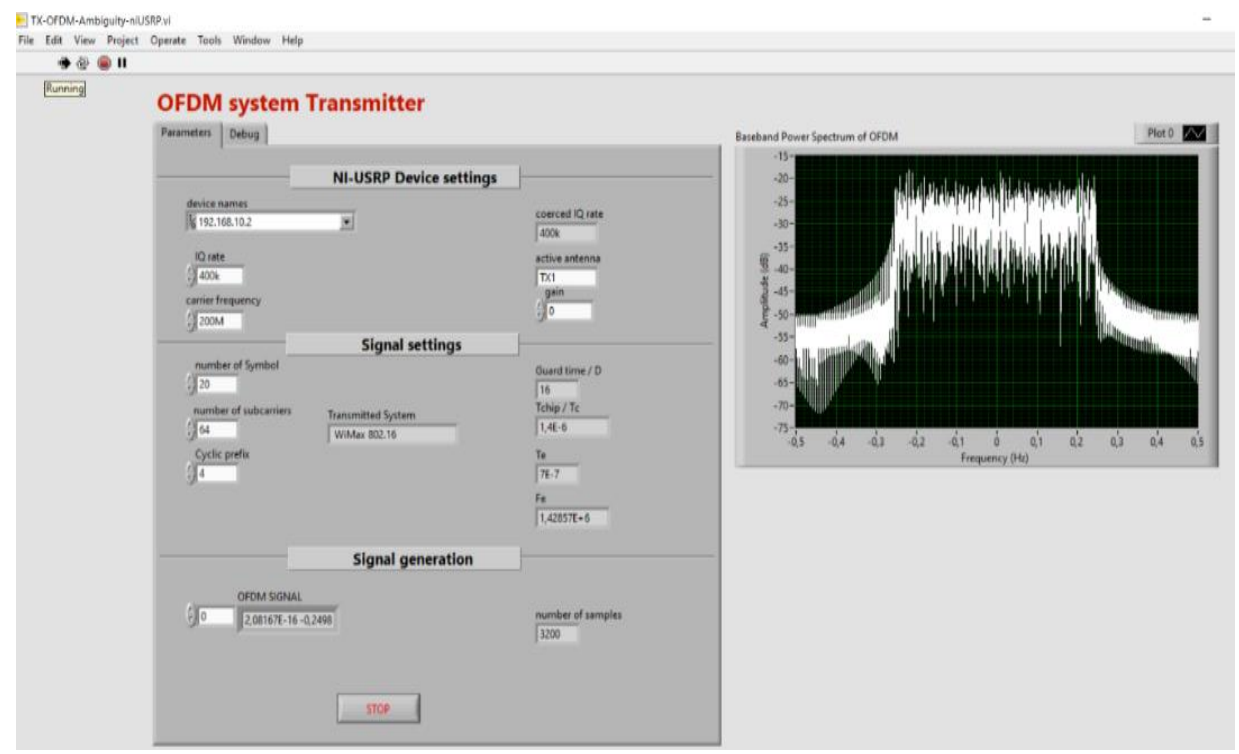

Figure 4. Transmitter VI front panel for OFDM signal

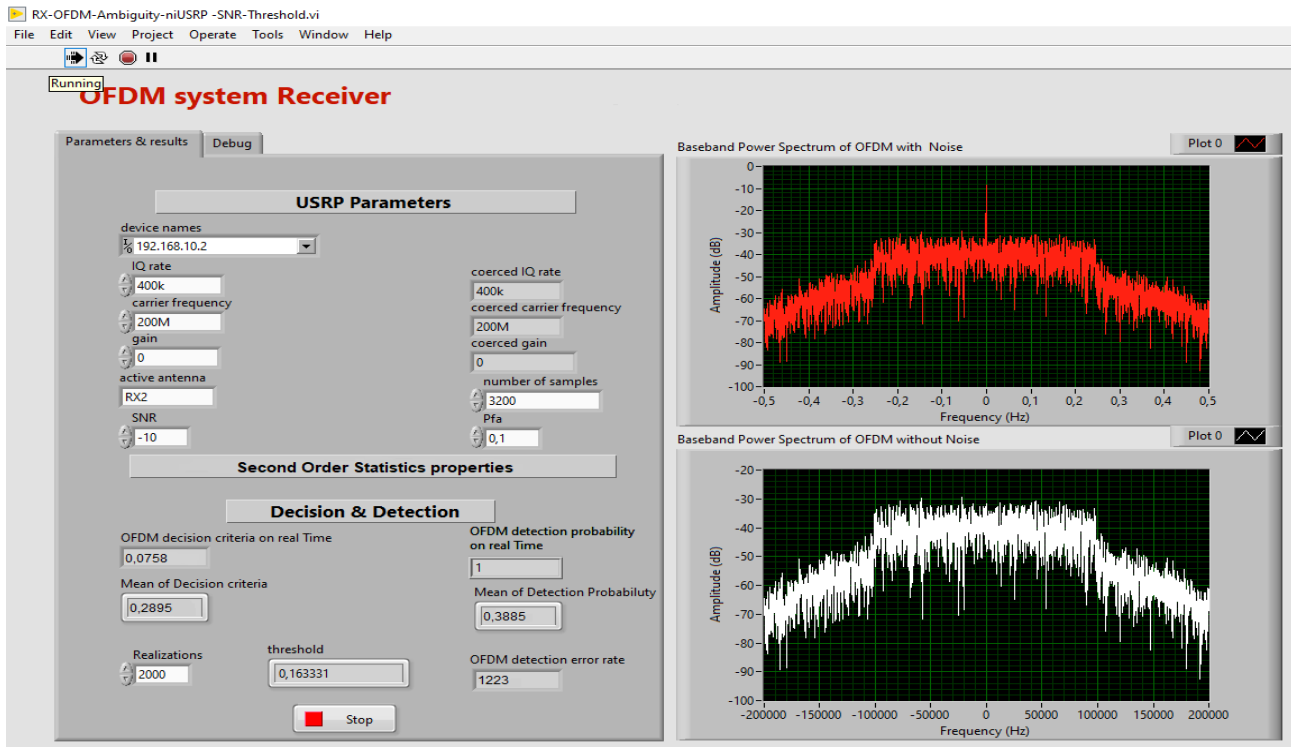

Figure 5. Receiver VI front panel for OFDM signal

\subsection{Parameters}

The following Tables 1 and 2 provide the characteristics of the USRP Software-defined radio and the parameters used in the MATLAB simulation and the USRP implementation: 
Table 1. NI USRP-2930 characteristics

\begin{tabular}{|c|c|}
\hline Parameter & Value \\
\hline Frequency Range & $50 \mathrm{MHz}$ to $2.2 \mathrm{GHz}$ \\
\hline Software Adjustable Frequency & $<1 \mathrm{kHz}$ \\
\hline Step & \\
\hline Gain Range & $0 \mathrm{~dB}$ to $31 \mathrm{~dB}$ \\
\hline $\begin{array}{l}\text { Software Adjustable Output } \\
\text { Power Step }\end{array}$ & $1.0 \mathrm{~dB}$ \\
\hline $\begin{array}{l}\text { Number of antenna connection } \\
\text { ports }\end{array}$ & 3: GPS, TX1/RX1, RX2 \\
\hline Maximum Input Power $\left(\mathrm{P}_{\text {in }}\right)$ & $0 \mathrm{dBm}$ \\
\hline Maximum Output Power $\left(\mathrm{P}_{\text {out }}\right)$ & $15 \mathrm{dBm}$ to $20 \mathrm{dBm}$ \\
\hline $\begin{array}{l}\text { Digital to Analog Conversion } \\
\text { (DAC) }\end{array}$ & $\begin{array}{l}2 \text { channels, } 400 \mathrm{MS} / \mathrm{s}, 16 \\
\text { bits }\end{array}$ \\
\hline $\begin{array}{l}\text { Analog to Digital Conversion } \\
\text { (ADC) }\end{array}$ & $\begin{array}{l}2 \text { channels, } 100 \mathrm{MS} / \mathrm{s}, 14 \\
\text { bits }\end{array}$ \\
\hline Instantaneous Real-Time & $20 \mathrm{MHz}$ (16-bit samples), \\
\hline Bandwidth & $40 \mathrm{MHz}$ (8-bit samples) \\
\hline
\end{tabular}

Table 2. Simulation and implementation parameters

\begin{tabular}{ll}
\hline \multicolumn{1}{c}{ Parameter } & \multicolumn{1}{c}{ Value } \\
\hline IQ Rate & $400 \mathrm{k}$ \\
Carrier frequency & $200 \mathrm{MHz}$ \\
Gain & $0 \mathrm{~dB}$ \\
Active antennas & TX1/RX1 \\
Number of Symbols (K) & 20 \\
Number of Subcarriers & 64 \\
$(\mathrm{~N})$ & \\
Cyclic Prefix $\left(D T_{c}\right)$ & 4 \\
Transmitted System & $3 \mathrm{GPP}(\mathrm{LTE})$, WiMAX \\
& (IEEE 802.16), DVB-T \\
& $2 \mathrm{~K}$, IEEE 802.22-1K, \\
& IEEE 802.22-2K, IEEE \\
Pfa & $802.22-4 \mathrm{~K}$ \\
SNR Range & 0.1 \\
Number of realizations & $-20 \mathrm{~dB}$ to $10 \mathrm{~dB}$ \\
\end{tabular}

\section{RESULTS AND DISCUSSION}

In this section, we display the experimental results of the implementation of OFDM signal recognition based on the correlation approach using the NI USRP-2930 platform, the displayed results are averaged over 2000 realizations. We have generated an OFDM signal randomly from six OFDM standards, 3GPP(LTE), WiMAX (IEEE 802.16), DVB-T 2K, IEEE 802.22-1K, IEEE 802.22-2K, and IEEE 802.22-4K. The signal is modulated by $N=64$ subcarriers uncoded QPSK, the length of the cyclic prefix $(C P)$ is fixed at 4 with $K=20$ symbols available at the OFDM receiver. In practice, the performance of our method can only be observed through the detection value below the threshold $\eta$ depending of $S N R$ and $P_{f a}$ parameters given by (9) in order to distinguish an OFDM signal from another type of digital signal. The chip duration $T_{c}$ of OFDM symbol for each standard system is stocked in our database.

In Figure 6, we plot the decision criteria versus the SNR for OFDM signals of the six standards mentioned above. As we can notice when approaching to $S N R=-2 d B$, the decision criteria tends towards 0 , which shows the good performance of the proposed method to detect the OFDM signal successfully. Moreover, the MATLAB simulation results and the USRP implementation measurements are closer to each other.

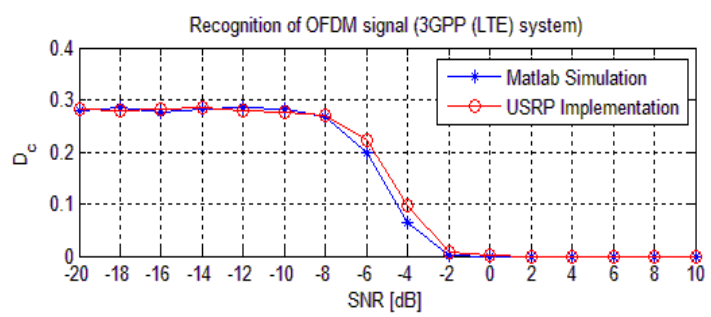

Recognition of OFDM signal (DVBT-2K system)

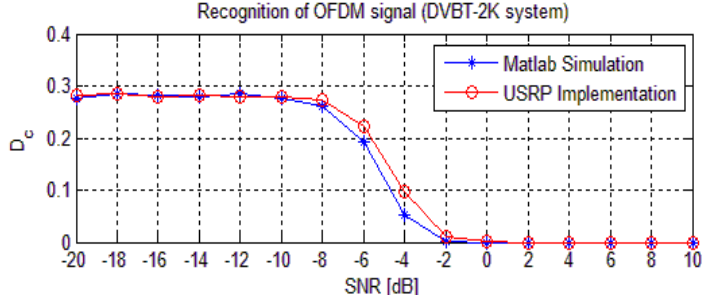

Recognition of OFDM signal (IEEE 802.22-2K) system)

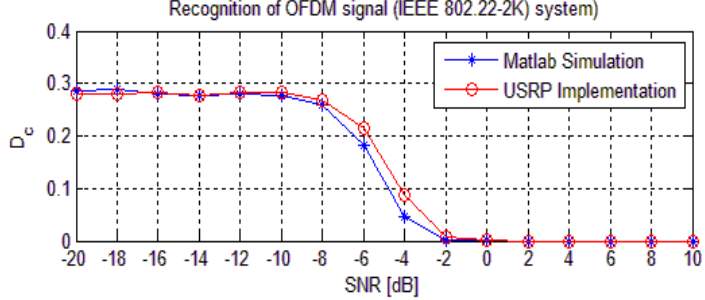

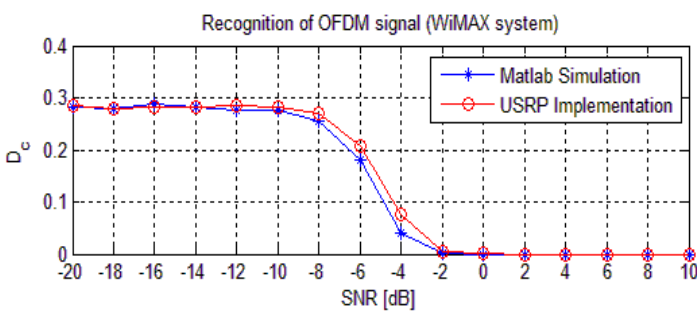

Recognition of OFDM signal (IEEE 802.22-1K system)

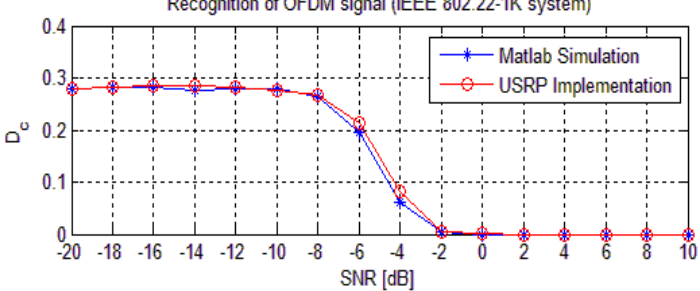

Recognition of OFDM signal (IEEE 802.22-4K) system)

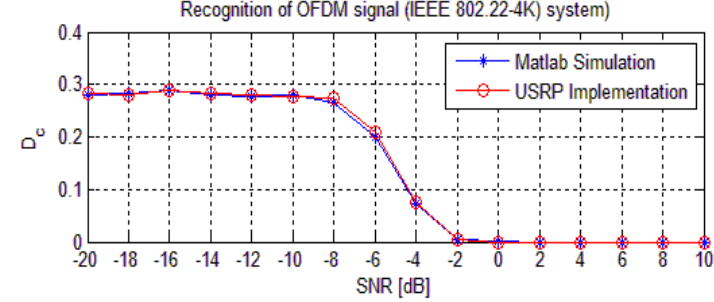

Figure 6. Detection criteria $\left(D_{c}\right)$ vs. SNR for OFDM signals 
Figure 7 illustrates the probability of detection versus SNR for the six standards based on OFDM modulation. We plot the detection probability defined by $P_{D}=P\left(D_{c}<\eta \mid O F D M\right)$, where $D_{c}$ is the decision criteria (9) and $\eta$ is the threshold (10). We notice that our method demonstrates always a strong performance to detect OFDM signals among other digital signals in a systematic and intelligent way even with low SNR values. We can also see as illustrated in the figures that the curves obtained by MATLAB simulation and implementation measurements on the USRP software defined radio have the same behavior and are more closely related to each other.
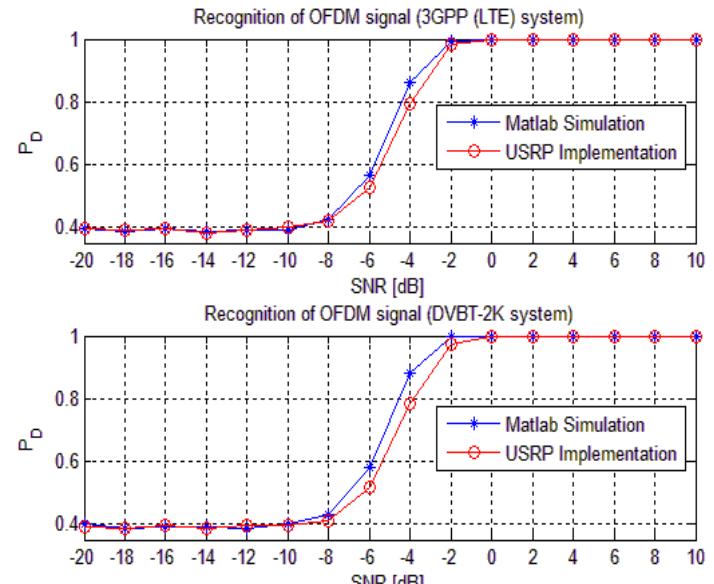

Recognition of OFDM signal (IEEE 802.22-2K) system)

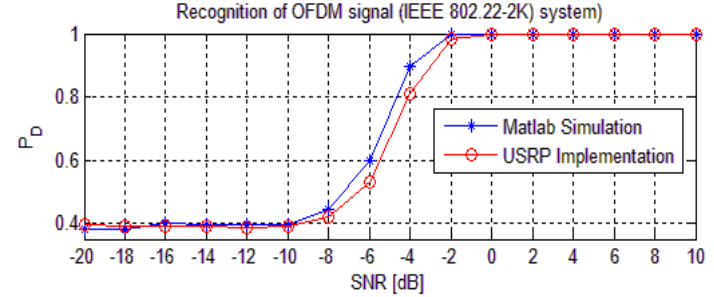

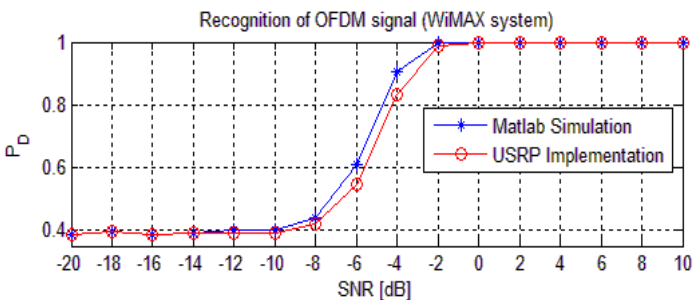

Recognition of OFDM signal (IEEE 802.22-1K system)

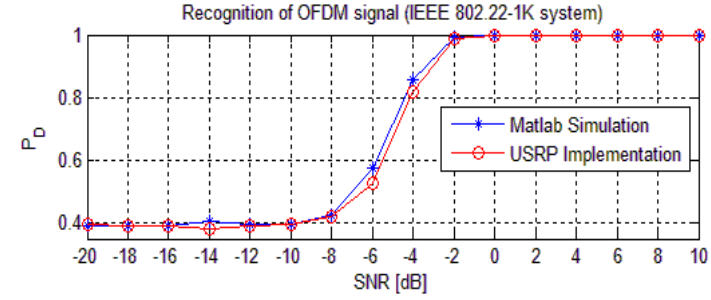

Recognition of OFDM signal (IEEE 802.22-4K) system)

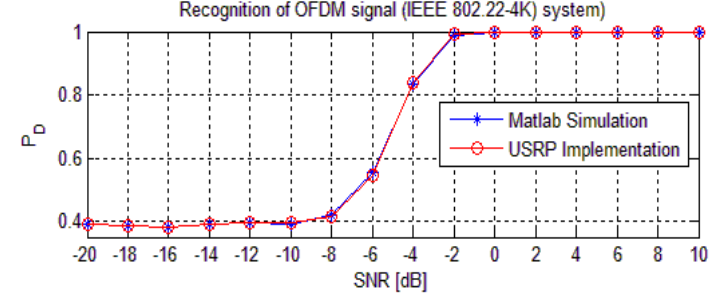

Figure 7. Detection probability $\left(\mathrm{P}_{\mathrm{D}}\right)$ of recognition OFDM systems vs. SNR

\section{CONCLUSION}

The proposed method described in this paper allows us to recognize OFDM signal used by the wireless standards present in a Radio Frequency (RF) receiver by using only a particular property of the second-order statistics. We have analyzed mathematically and numerically through a series of equations and simulation results that illustrate the efficiency and performance of the proposed method. Compared to the literature, we have also considered more realistic situations since several recognition methods for wireless communication systems have been presented considering only synthetic models. For that, we have set up a test platform based on the USRP software defined radio to generate real OFDM signals. The performance of our method is illustrated by a real implementation using NI USRP-2930 hardware device, which provides good experimental results that are closer to those of Monte Carlo simulations executed by MATLAB software. Moreover, the method shows that it is absolutely robust and more efficient in low SNR values. The future work will be to propose a method to discriminate standards between them. We will also study the contribution of artificial intelligence in the context of cognitive radio.

\section{REFERENCES}

[1] M. Alibakhshikenari, et al., "A Comprehensive Survey of "Metamaterial Transmission-Line Based Antennas: Design, Challenges, and Applications," in IEEE Access, vol. 8, pp. 144778-144808, 2020, doi: 10.1109/ACCESS.2020.3013698.

[2] P. Diego and C. Hernandez, "Cognitive radio for TVWS usage," TELKOMNIKA Telecommunication, Computing, Electronics and Control, vol. 17, no. 6, pp. 2735-2746, December 2019, doi: 10.12928/telkomnika.v17i6.13111.

[3] J. Mitola, "Cognitive Radio: An Integrated Agent architecture for Software Defined Radio," Phd thesis, Royal Institute of Technology (Stockholm, Sweden), 2000. 
[4] S. Haykin, "Cognitive radio: Brain-empowered wireless communications," IEEE Journal on Selected Areas in Communications, Special Issue on Cognitive Networks, vol. 23, pp. 201-220, Feb. 2005, doi: 10.1109/JSAC.2004.839380.

[5] T. Yucek and H. Arslan, "A survey of spectrum sensing algorithms for cognitive radio applications," IEEE Communications Surveys and Tutorials, vol. 11, no. 1, pp. 116-130, 2009, doi: 10.1109/SURV.2009.090109.

[6] D. Bao, L. De Vito, and S. Rapuano, "A histogram-based segmentation method for wideband spectrum sensing in cognitive radios," IEEE Transactions on Instrumentation and Measurement, vol. 62, no. 7, pp. 1900-1908, 2013, doi: 10.1109/TIM.2013.2251821.

[7] E. Axell, G. Leus, E. G. Larsson, and H. V. Poor, "Spectrum sensing for cognitive radio: State of the art and recent advances," IEEE Signal Processing Magazine, vol. 29, no. 3, pp. 101-116, 2012, doi: 10.1109/MSP.2012.2183771.

[8] F.-Z. El Bahi, H. Ghennioui and M. Zouak, "Spectrum sensing technique of OFDM signal under noise uncertainty based on Mean Ambiguity Function for cognitive radio," Physical Communication, vol. 33, pp. 142-150, April 2019, doi: 10.1016/j.phycom.2018.12.022.

[9] R. Deepa and Y. Ravinder, "A statistical approach to spectrum sensing using bayes factor and p-Values," International Journal of Electrical and Computer Engineering (IJECE), vol. 9, no. 4, pp. 2910-2917, August 2019, doi: 10.11591/ijece.v9i4.pp2910-2917.

[10] S. Dikmese, S. Srinivasan, and M. Renfors, "FFT and Filter Bank Based Spectrum Sensing and Spectrum Utilization for Cogntive Radios," 2012 5th International Symposium on Communications, Control and Signal Processing, 2012, pp. 1-5, doi: 10.1109/ISCCSP.2012.6217788.

[11] J. A. C. Bingham, "Multicarrier modulation for data transmission: an idea whose time has come," IEEE Commull. Mag., vol. 28, no. 5, pp. 5-14, 1990, doi: 10.1109/35.54342.

[12] R. V. Nee and R. Prasad, "OFDM for Wireless Multimedia Communications," Artech House, 2000.

[13] IEEE Computer Society LAN MAN Standards Committee, "Wireless LAN medium access control (MAC) and physical layer (PHY) specifications," ANSI/IEEE Std. 802.11-1999, 1999.

[14] Local and Metropolitan Area Networks-Part 16, Air Interface for Fixed Broadband Wireless Access Systems, IEEE Standard IEEE 802.160-2001.

[15] B. Wang and L. Ge, "A novel algorithm for identification of OFDM signal," Proceedings. 2005 International Conference on Wireless Communications, Networking and Mobile Computing, 2005, pp. 261-264, doi: 10.1109/WCNM.2005.1544031.

[16] D. Grimaldi, S. Rapunao, and G. Truglia, "An automatic digital modulation classifier for measurement on telecommunication networks," IEEE Transactions on Instrumentation and Measurement, pp. 957-962, 2002, doi: 10.1109/TIM.2007.895675.

[17] W. Akmouche, "Detection of multicarrier modulations using 4th order cumulants," in Proc. IEEE MILCOM, 1999, pp. 432-436, doi: 10.1109/MILCOM.1999.822720.

[18] P. S. Thakur, S. Madan, and M. Madan, "Automatic Classification of WiMAX Physical Layer OFDM Signals Using Neural Network," Next-Generation Networks, 2018.

[19] A. Bouzegzi, P. Ciblat and P. Jallon, "New algorithms for blind recogni- tion of OFDM based systems," Signal Processing, vol. 90, no. 3, pp. 900-913, 2010, doi: 10.1016/j.sigpro.2009.09.017.

[20] F.-X. Socheleau, S. Houcke, P. Ciblat, and A. Aïssa-El-Bey, "Cognitive OFDM system detection using pilot tones second and third-order cyclostationarity," Signal Process, vol. 91, no. 2, pp. 252-268, Feb. 2011, doi: 10.1016/j.sigpro.2010.07.003.

[21] P. D. Sutton, K. E. Nolan, and L. E. Doyle, "Cyclostationary signatures in practical cognitive radio applications," IEEE J. Sel. Areas Commun., vol. 26, no. 1, pp. 13-24, Jan. 2008, 10.1109/JSAC.2008.080103.

[22] F.-X. Socheleau, P. Ciblat, and S. Houcke, "OFDM system identification for cognitive radio based on pilot-induced cyclostationarity," 2009 IEEE Wireless Communications and Networking Conference, 2009, pp. 1-6, doi: 10.1109/WCNC.2009.4917840.

[23] A. Al-Habashna, O. A. Dobre, R. Venkatesan, and D. C. Popescu, "Second-order cyclostationarity of mobile WiMAX and LTE OFDM signals and application to spectrum awareness in cognitive radio systems," IEEE J. Sel. Topics Signal Process., vol. 6, no. 1, pp. 26-42, Feb. 2012, doi: 10.1109/JSTSP.2011.2174773

[24] W. Jerjawi, Y. A. Eldemerdash, and O. A. Dobre, "Second-order cyclostationarity-based detection of LTE SCFDMA signals for cognitive radio systems," IEEE Transactions on Instrumentation and Measurement, vol. 64, no. 3, pp. 823-833, Mar. 2015, doi: 10.1109/TIM.2014.2357592.

[25] K. Tekbiyik, H. Tuğrel, and G. K. Karabulut, and G. Ayyildiz, "Blind recognition of OFDM signals based on cyclostationary signal analysis," 24th International Conference on Telecommunications (ICT), 2017, pp. 1-5, 10.1109/ICT.2017.7998230

[26] M. Firdaoussi, H. Ghennioui, and M. El Kamili," New algorithm for blind recognition of OFDM based systems using Second-Order Statistics," in Processing 2015 IEEE WINCOM, pp. 1-4, 2015, doi: 10.1109/WINCOM.2015.7381336.

[27] M. Oner and F. Jondral, "On the extraction of the channel allocation information in spectrum pooling systems," IEEE Journal on Selected Areas in Communications, vol. 25, no. 3, pp. 558-565, Apr. 2007, doi: 10.1109/JSAC.2007.070406.

[28] National Instruments Co.," USRP-2930 Datasheet," [Online]. Available: https://www.ni.com/pdf/manuals/375987d.pdf. Accessed 07 November 2020. 


\section{BIOGRAPHIES OF AUTHORS}

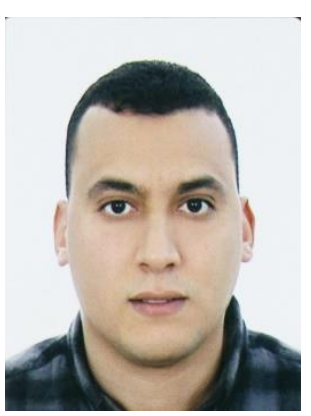

Mohamed Firdaoussi is actually working to get a Ph.D. degree in the laboratory of Applied Physics, Computer Science and Statisitics, in Faculty of Sciences Dhar EL Mehraz of Fez at Sidi Mohamed Ben Abdelah University, Morocco. $\mathrm{He}$ is an engineer in Network and Telecommunication, graduated in 2010 from National School of Applied Sciences of Fez, Morocco. His main research interests are signal processing, cognitive radio and network security.

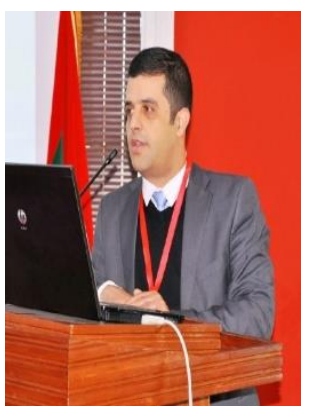

Hicham Ghennioui is an assistant director of Signals, Systems and Components Laboratory at Faculty of Sciences and Technologies, Fez, Morocco. Since 2011, he is a full-time Associate Professor at the Faculty of Sciences and Technologies, Fez. He received the Ph.D degree in Computer Science and Telecommunications in 2008, jointly from Mohamed V University and the Toulon University. In 2004, received the D.E.S.A. degree in Computer Science and Telecommunications from the Mohamed V University. His main research interests are signal/image processing including blind sources separation, data analytic, deblurring and cognitive radio.

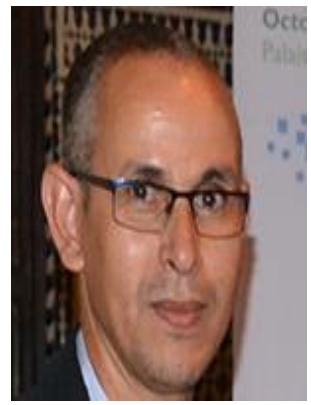

Mohamed El Kamili received the Ph.D. degree in Computer Science and Operations Research from the Mohammed V University, Rabat, Morocco. He have co-authored many journal articles and book chapters, and many conference publications. He acts as a reviewer for professional publications, prestigious international journals and international conferences, as IEEE Network Magazine, computer communication journal (ComCom), IEEE ICC, IEEE Globcom conference, IEEE IWCMC, IEEE WCNC, ADHOCNET. Currently, he is a full-time Associate Professor in Computer Networks at Computer Engineering Department, Higher School of Technology, Hassan II University of Casablanca, Morocco. He is a permanent member of the research team "Big Data \& Internet of Skills" of the "Computer Science \& Smart Systems (C3S) Laboratory". His current research interests include Networking Games, Design of communication protocols for wireless networks, Wireless MAC protocols design and evaluation, intelligent wireless networks and learning algorithms, Cognitive radio and Delay Tolerant Network, IoT and D2D communications. Dr. EL KAMILI is a founder and the president of the Moroccan Mobile Computing and Intelligent Embedded-Systems Society (Mobitic), http://www.mobitic.org/. He has also co-founded the International Conference on Wireless Networks and Mobile Communications (WINCOM, http://www.wincom-conf.org) technically support by IEEE ComSoc. Dr. El Kamili acts as a local chair of the fourth edition of WINCOM that held in fez Morocco, October 26-29, 2016, and he acts as a Vice-General Chair for the seventh edition of WINCOM, October 29 -November 1, 2019. During January 2017 to march 2020, He was a member of the IEEE Morocco Section committee; he has occupied the position of Conference Coordinator

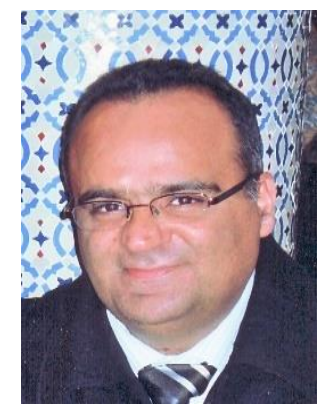

Mohamed Lamrini received the PhD degree from the Claude Bernard -Lyon University in 1993. He is currently a professor of computer science at USMBA-Fez University. He is also a member of the LPAIS Laboratory. His research interests include software quality (CMMI, Six sigma, ISO 9001), Industrial Engineering (Methods and statistical tools). 